\title{
Monte Carlo simulations of a supersymmetric matrix model of dynamical compactification in non perturbative string theory
}

\author{
Konstantinos N. Anagnostopoulos ${ }^{* *}$ \\ Physics Department, National Technical University of Athens \\ Zografou Campus, 157-80 Zografou, Greece \\ E-mail: konstantemail.ntua.ar
}

\section{Takehiro Azuma}

Institute for Fundamental Sciences, Setsunan University, 17-8 Ikeda Nakamachi, Neyagawa, Osaka 572-8508, Japan

E-mail: azuma@mpg.set.sunan.ac.jp

\section{Jun Nishimura}

High Energy Accelerator Research Organization (KEK) and Graduate University for Advanced Studies (SOKENDAI), 1-1 Oho, Tsukuba 305-0801, Japan

E-mail: inishidpost.kek.jp

The IKKT or IIB matrix model has been postulated to be a non perturbative definition of superstring theory. It has the attractive feature that spacetime is dynamically generated, which makes possible the scenario of dynamical compactification of extra dimensions, which in the Euclidean model manifests by spontaneously breaking the $\mathrm{SO}(10)$ rotational invariance (SSB). In this work we study using Monte Carlo simulations the 6 dimensional version of the Euclidean IIB matrix model. Simulations are found to be plagued by a strong complex action problem and the factorization method is used for effective sampling and computing expectation values of the extent of spacetime in various dimensions. Our results are consistent with calculations using the Gaussian Expansion method which predict SSB to SO(3) symmetric vacua, a finite universal extent of the compactified dimensions and finite spacetime volume.

The 30th International Symposium on Lattice Field Theory

June 24 29, 2012

Cairns, Australia

\footnotetext{
* Speaker.

$\dagger$ The work of K.N.A. was partially funded by the National Technical University of Athens through the Basic Research Support Programmes 2009 and 2010. The work of T.A. and J.N. is supported in part by Grant-in-Aid for Scientific Research (No. 23740211 for T.A. and 20540286, 23244057 for J.N.) from Japan Society for the Promotion of Science.
} 


\section{Introduction}

Superstring theory is a natural candidate of a unified theory of all interactions, including gravity. It lacks a non-perturbative definition, which would allow us to address dynamically questions such as the preferred vacuum where our universe sits and determine its properties such as classical spacetime dimensionality. The large $N$ limit of the 10 dimensional IKKT or IIB matrix model has been proposed to provide such a definition [四]. Using dualities, which suggest that the five types of superstring theory are actually limits of a unique theory, the IIB matrix model is expected to describe the unique underlying theory despite its explicit connection to perturbative type IIB superstring theory.

The IIB matrix model has a series of attractive features: The model has a unique scale, which raises the possibility for the theory to choose a unique vacuum. Spacetime and matter content can arise dynamically from the distribution of the eigenvalues of the bosonic matrices, which makes possible the realization of the scenario of dynamical compactification of extra dimensions. The motivation for studying such a scenario in the Euclidean model comes from noticing that lower dimensional configurations are stationary points of the fluctuating complex part of the action of the model [Q] ]. Using the Gaussian Expansion Method (GEM), one can show that the $\mathrm{SO}(3)$ symmetric vacuum has the smallest free energy density compared to higher or lower dimensional configurations [B] . Dynamical compactification, therefore, occurs by spontaneously breaking the $\mathrm{SO}(10)$ rotational symmetry of the model (SSB). Moreover, a universal scale $r$ for the small dimensions is computed $^{1}$, with the scale $R$ of the large dimensions being such that $R^{d} r^{10-d}=\ell^{10}$, with $\ell$ being the scale of the size of the symmetric configurations. The former relation is interpreted as a constant volume property of the $d$ dimensional (metastable) vacua of the model and leads to the conclusion that spacetime in the Euclidean IIB model has finite volume [B]].

Monte Carlo simulations of the Euclidean IIB matrix model, as well as simpler related models, can confirm these results from first principles and shine light into the mechanism of dynamical compactification of extra dimensions [ [ 9 , []]. Such simulations are hindered by a severe complex action problem and are quite difficult. Some results, however, have been obtained by studying related lower dimensional toy models using the so called factorization method. This method, proposed originally in [焑] has been tested also in Random Matrix theory and finite density QCD [四]. It attempts to sample the most important configurations that contribute to the partition function, first by numerically solving equations that compute the maxima of the distribution functions of appropriately chosen observables that are strongly correlated to the fluctuating phase [ [ ] ] and then by simulating a constrained system in the region of the solutions. This makes importance sampling possible by also taking into account the suppression of configurations by the fluctuations of the phase, together with the suppression caused by the measure and the real part of the action in the partition function. The overlap problem is thus solved and the complex action problem reduced due to milder fluctuations of the phase within the sampled configurations and the use of scaling properties that permit extrapolations to larger systems.

In this work, we present Monte Carlo simulation results of a 6 dimensional version of the IIB Matrix Model. Contrary to [圆, where the oneloop approximation was used, the full model is simulated. This is necessary since, as we will show, the short distance non perturbative dynamics

\footnotetext{
${ }^{1}$ Note that the actual length of each dimension is, by definition, $r^{1 / 2}$ and $R^{1 / 2}$ respectively.
} 
of the eigenvalues of the matrices play a crucial role in generating the scales of dynamical compactification. We show that in the absence of the complex part of the action, no SSB of the SO(6) rotational invariance occurs. We apply the factorization method in order to compute the eigenvalues $\lambda_{1}>\lambda_{2}>\ldots>\lambda_{6}$ of spacetime's "moment of inertia" tensor $T_{\mu \nu}$ and compare our results with those obtained in [3] using the GEM. In particular, we test the GEM predictions of SSB to SO(3) symmetric vacua, a universal scale $r$ for compactified dimensions and finite spacetime volume. Our results are consistent with these scenaria.

\section{The Model}

We study a 6 dimensional version of the IIB matrix model defined by the partition function

$$
Z=\int d A d \psi d \bar{\psi} \mathrm{e}^{-S_{b}-S_{f}}
$$

where $S_{b}=-\frac{N}{4} \operatorname{tr}\left[A_{\mu}, A_{v}\right]^{2}$ and $S_{f}=\frac{N}{2} \operatorname{tr} \bar{\psi}_{\alpha}(\Gamma)_{\alpha \beta}\left[A_{\mu}, \psi_{\beta}\right]$. The model can be formally obtained by the dimensional reduction of the Euclidean 6 dimensional $\mathscr{N}=1 \mathrm{U}(N)$ Super Yang Mills theory to zero dimensions: The $N \times N$ matrices $A_{\mu}, \mu=1, \ldots, 6$ are traceless and hermitian and transform as $\mathrm{O}(6)$ vectors. The $N \times N$ matrices $\psi_{\alpha}, \bar{\psi}_{\alpha}$ are traceless with grassmannian entries and transform as Weyl spinors. The model turns out to have $\mathscr{N}=2$ supersymmetry, which leads to the interpretation of the eigenvalues of $A_{\mu}$ defining the points of the 6 dimensional (Euclidean) spacetime $^{2}$ [四]. Dynamical compactification can manifest if the distribution of these points break $\mathrm{O}(6)$ rotational invariance spontaneously (SSB). The order parameters of such SSB are the expectation values $\left\langle\lambda_{1}\right\rangle, \ldots,\left\langle\lambda_{6}\right\rangle$ of the "moment of inertia tensor" of spacetime $T_{\mu \nu}=\frac{1}{N} \operatorname{tr} A_{\mu} A_{\nu}$, where the eigenvalues $\lambda_{1}>\ldots>\lambda_{6}$ are ordered before taking the expectation value. SSB occurs if, in the large $N$ limit, some of the expectation values $\left\langle\lambda_{1}\right\rangle, \ldots,\left\langle\lambda_{d}\right\rangle$ grow "large" and the remaining $\left\langle\lambda_{d+1}\right\rangle, \ldots,\left\langle\lambda_{6}\right\rangle$ remain small. The large eigenvalues define the extended dimensions of spacetime and we obtain dynamically a $d$ dimensional spacetime. This scenario has been studied using GEM in [3] where $\mathrm{SO}(3)$ invariant configurations were found to have the minimum free energy density, thus dominating the path integral (2. have a $d$-independent thickness $r^{1 / 2}$, whereas the $d$ large ones have thickness $R^{1 / 2}$, such that

$$
R^{d} r^{6-d}=\ell^{6}
$$

where $\ell \approx 0.627$ is the square of the extent of the $\mathrm{SO}(6)$ symmetric configurations, $\left\langle\lambda_{1}\right\rangle=\ldots=$ $\left\langle\lambda_{d}\right\rangle=R$ and $\left\langle\lambda_{d+1}\right\rangle=\ldots=\left\langle\lambda_{6}\right\rangle=r$. The $\mathrm{SO}(6)$ symmetric configurations dominate in the phase quenched model discussed below where SSB does not occur [5]. Eq. (2.2) expresses the constant volume property of the $\mathrm{SO}(d)$ vacua and implies that the volume of spacetime in the 6 dimensional Euclidean IIB matrix model is finite [3].

Monte Carlo simulations are performed by integrating out the fermions in (2.] first, obtaining

$$
Z=\int d A \operatorname{det} \mathscr{M} \mathrm{e}^{-S_{b}}=\int d A \mathrm{e}^{-S_{0}+i \Gamma},
$$

\footnotetext{
${ }^{2}$ Albeit a non classical spacetime with a fuzzy geometry since the dominant configurations of $A_{\mu}$ cannot be simultaneously diagonalizable [可.
} 
where $\operatorname{det} \mathscr{M}=Z_{f}[A]=\int d \psi d \bar{\psi} \mathrm{e}^{-S_{f}}$ and $S_{0}=S_{b}-\log |\operatorname{det} \mathscr{M}|$. The determinant det $\mathscr{M}=$ $|\operatorname{det} \mathscr{M}| \mathrm{e}^{i \Gamma}$ turns out to be generically complex creating a very strong complex action problem in the simulations. We applied the factorization method [G], which first amounts to considering the phase quenched model $Z_{0}=\int d A \mathrm{e}^{-S_{0}}$. In this model, we computed the phase quenched expectation values $\left\langle\lambda_{1}\right\rangle_{0}, \ldots,\left\langle\lambda_{n}\right\rangle_{0}$, and defined the normalized eigenvalues $\tilde{\lambda}_{n}=\lambda_{n} /\left\langle\lambda_{n}\right\rangle_{0}$. Deviation of $\left\langle\tilde{\lambda}_{n}\right\rangle$ from 1 , is the result of the suppression of the dominant configurations of $Z_{0}$ by the fluctuations of the phase $\Gamma$. Due to the strong correlations of $\lambda_{n}$ with $\Gamma$, we consider the distribution functions [圆, 目]

$$
\rho_{n}(x)=\left\langle\delta\left(x-\tilde{\lambda}_{n}\right)\right\rangle=\frac{1}{C} \rho_{n}^{(0)}(x) w_{n}(x),
$$

where $^{3} \rho_{n}^{(0)}(x)=\left\langle\delta\left(x-\tilde{\lambda}_{n}\right)\right\rangle_{0}, w_{n}(x)=\left\langle\mathrm{e}^{i \Gamma}\right\rangle_{n, x}$ and $\langle\cdot\rangle_{n, x}$ denotes expectation values within the constrained system $Z_{n, x}=\int d A \mathrm{e}^{-S_{0}} \delta\left(x-\tilde{\lambda}_{n}\right)$.

The value of $\left\langle\lambda_{n}\right\rangle$ in the large $N$ limit is determined by the minimum of the "free energy" $\mathscr{F}_{n}(x)=-\log \rho_{n}(x)$ which, given Eq. (2.4) , is the large $N$ limit of a solution to

$$
\frac{1}{N^{2}} f_{n}^{(0)}(x) \equiv \frac{1}{N^{2}} \frac{d}{d x} \log \rho_{n}^{(0)}(x)=-\frac{1}{N^{2}} \frac{d}{d x} \log w_{n}(x) .
$$

Using the dominant solution of ([2.5) as an estimator of $\left\langle\lambda_{n}\right\rangle$ for finite $N$, has the advantages that the overlap problem is solved by simulating $Z_{n, x}$ and the complex action problem reduced, since $\Phi_{n}(x)=\lim _{N \rightarrow \infty} \frac{1}{N^{2}} \log w_{n}(x)$ is found to scale for relatively small $N$ and it is possible to extrapolate solutions to larger values of $N$. Moreover, the numerical errors of the solution do not grow exponentially with $N$ [四, 四].

\section{Simulations and Results}

Monte Carlo simulations are performed on the system $Z_{n, V}=\int d A \mathrm{e}^{-S_{0}-V\left(\lambda_{n}\right)}$ where $V(z)=$ $\frac{1}{2} \gamma(z-\xi)^{2}$ and $\gamma, \xi$ are parameters. The rational hybrid Monte Carlo method (RHMC) is used in the simulations. We take $\gamma$ large enough, so that $\rho_{n, V}(x)$ is sharply peaked at $x_{p}$ and the results are independent of $\gamma$. We use the estimators $x_{p}=\left\langle\tilde{\lambda}_{n}\right\rangle_{n, V}, w_{n}\left(x_{p}\right)=\left\langle\mathrm{e}^{i \Gamma}\right\rangle_{n, V}$ and $f_{n}^{(0)}\left(x_{p}\right)=$ $\left\langle\lambda_{n}\right\rangle_{0} V^{\prime}\left(\left\langle\lambda_{n}\right\rangle_{n, V}\right)$.

For $\gamma=0$ we obtain the phase quenched model $Z_{0}$. We simulate this system and show the results for $\left\langle\lambda_{n}\right\rangle_{0}, n=1, \ldots, 6$ in the left plot of Fig. $\square$. In the large $N$ limit, these values converge to the same value $\ell \approx 0.627$ as predicted by GEM [B] . No SSB of $\mathrm{SO}(6)$ occurs in the absence of a fluctuating phase $\Gamma$ as expected [ [2, []].

In order to compute the solution of ([2.5) in the large $N$ limit, it is important to use the scaling properties of the functions $w_{n}(x)$ and $f_{n}^{(0)}(x)$ for small $x$ and in the large $N$ limit. We find that $\Phi_{n}(x)$, $n=3,4,5,6$ is almost constant for large $x$. Thus the extended direction becomes decorrelated with the phase and there is no need to constrain the large eigenvalues as in [ [ $]$ ]. We also observe good scaling of $\frac{1}{N^{2}} \log w_{n}(x)$ for $12<N<24$. For solving ([2.5), the small $x$ scaling behavior [ [ ] ]

\footnotetext{
${ }^{3}$ The constant $C=\left\langle\mathrm{e}^{i \Gamma}\right\rangle_{0}$ is not necessary in the calculations described below.
} 

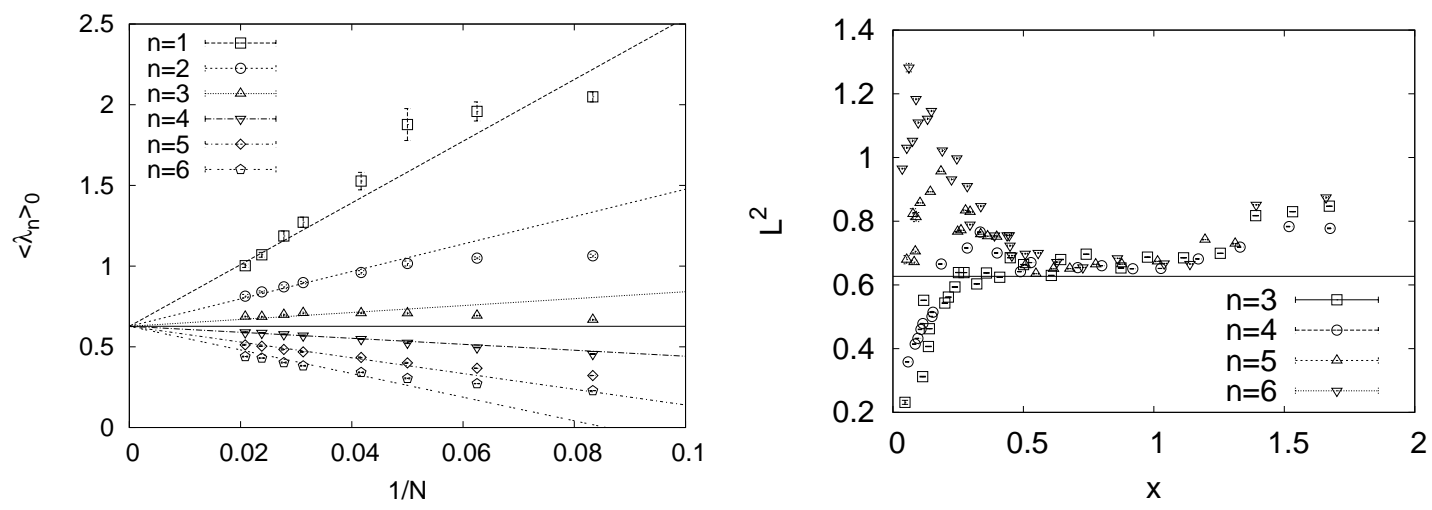

Figure 1: (LEFT) The $\left\langle\lambda_{n}\right\rangle_{0}$ of the phase quenched model are plotted against $1 / N$ for $12 \leq N \leq 48$. The horizontal line is $\ell=0.627$. $(\mathrm{RIGHT}) L_{n}^{2}=\left(\prod_{i=1}^{6}\left\langle\lambda_{i}\right\rangle_{n, V}\right)^{1 / 6}$ for $N=32$. The horizontal line is $\ell=0.627$.

$\frac{1}{N^{2}} \log w_{n}(x) \sim-c_{0, n}-c_{1, n} x^{7-n}, n=2, \ldots, 6$ is important. We compute the coefficients $c_{0, n}$ and $c_{1, n}$, as shown in the left plot of Fig. ㅁ. For $f_{n}^{(0)}(x)$ the expected small $x$ scaling is $\frac{1}{N^{2}} f_{n}^{(0)}(x) \sim$ $\left\{\frac{1}{2}(7-n)+2 \delta_{n 1}\right\} \frac{1}{x}$ and implies the existence of a hard core potential suppressing the small $x$ region, as it was first found in [5]. This is a strictly non perturbative effect which is absent e.g. in the one loop approximation[目]. Around $x=1 \mathrm{a} \frac{1}{N} f_{n}^{(0)}(x)$ scaling is found to dominate ${ }^{4}$. This adds finite size effects to the small- $x$ scaling which can be subtracted by removing the $\mathscr{O}(1 / N)$ term $g_{n}(x) / N x$, where we take $g_{n}(x)=a_{1, n}(x-1)+a_{2, n}(x-1)^{2}+a_{3, n}(x-1)^{3}$ around the peak $x \approx 1$. The computation of $g_{n}(x)$ is done by the fit in the right plot of Fig. $\square$, where we show the result for $n=4$. Finally, the left hand side of $(\underline{2.5})$ is estimated by the function $\frac{1}{N^{2}} f_{n}^{(0)}(x)-$ $\frac{g_{n}(x)}{N x}=\left\{\frac{1}{2}(7-n)+2 \delta_{n 1}\right\} \frac{\exp \left(-q_{n} x\right)}{x}$, where $q_{n}$ is determined by the fit of the left plot of Fig. [3. In the same plot we also show $-\Phi_{n}^{\prime}(x)$, and the intersection of the two curves estimates the solution of (2.5) for $n=4$. It is found that $\left\langle\tilde{\lambda}_{4}\right\rangle=0.31(2)$. The corresponding GEM prediction is $r / \ell \approx 0.223 / 0.627=0.355$ [B] . Preliminary results for $n=3,5$ and 6 yield similar results which are consistent with the GEM finding that $r$ takes a universal, $d$-independent value. The constant volume property is further studied by computing $L_{n}^{2}=\left(\prod_{i=1}^{6}\left\langle\lambda_{i}\right\rangle_{n, V}\right)^{1 / 6}$. The results are shown in the right plot of Fig. . For $0.5<x<1 L_{n}^{2} \approx \ell \approx 0.627$ as predicted by the GEM.

Finally we attempt to compare the free energies $\mathscr{F}_{3}(x)$ and $\mathscr{F}_{4}(x)$ by numerically computing $\Delta_{34}=-\mathscr{F}_{3}(x)+\mathscr{F}_{4}(x)$. Their value at the solution of Eq. ([2.5) compares the free energies of the $\mathrm{SO}(3)$ and $\mathrm{SO}(2)$ vacua respectively. The calculation is done as described in [8] by computing

$$
\Delta_{34}=\frac{1}{N^{2}} \log w_{3}\left(x_{s}\right)-\frac{1}{N^{2}} \log w_{4}\left(x_{s}\right)-\int_{\mathrm{SO}(2)}^{\mathrm{SO}(3)} \frac{1}{N^{2}} \frac{d}{d x} \log \rho^{(0)}(x) \simeq \frac{1}{N^{2}} \log w_{3}\left(x_{s}\right)-\frac{1}{N^{2}} \log w_{4}\left(x_{s}\right)
$$

\footnotetext{
${ }^{4}$ Note that in the one loop approximation, the $\frac{1}{N} f_{n}^{(0)}(x)$ scaling holds for all values of $x$ [䧃].
} 

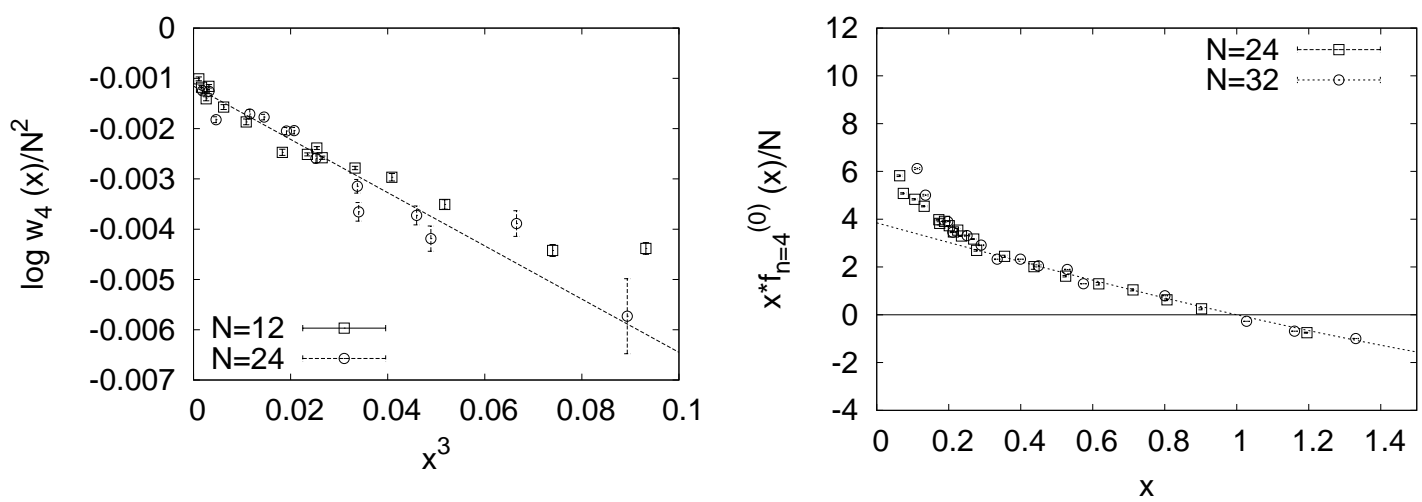

Figure 2: (LEFT) Small $x$ scaling of $\log w_{4}(x) / N^{2}$, according to the discussion on page $\square$ for $N=24$. (RIGHT) $\frac{1}{N} f_{4}^{(0)}(x)$ scaling around the peak $x \approx 1$, according to the discussion on page $\square$ for $N=24$. The fit is the function $g_{4}(x)$ described in the text.
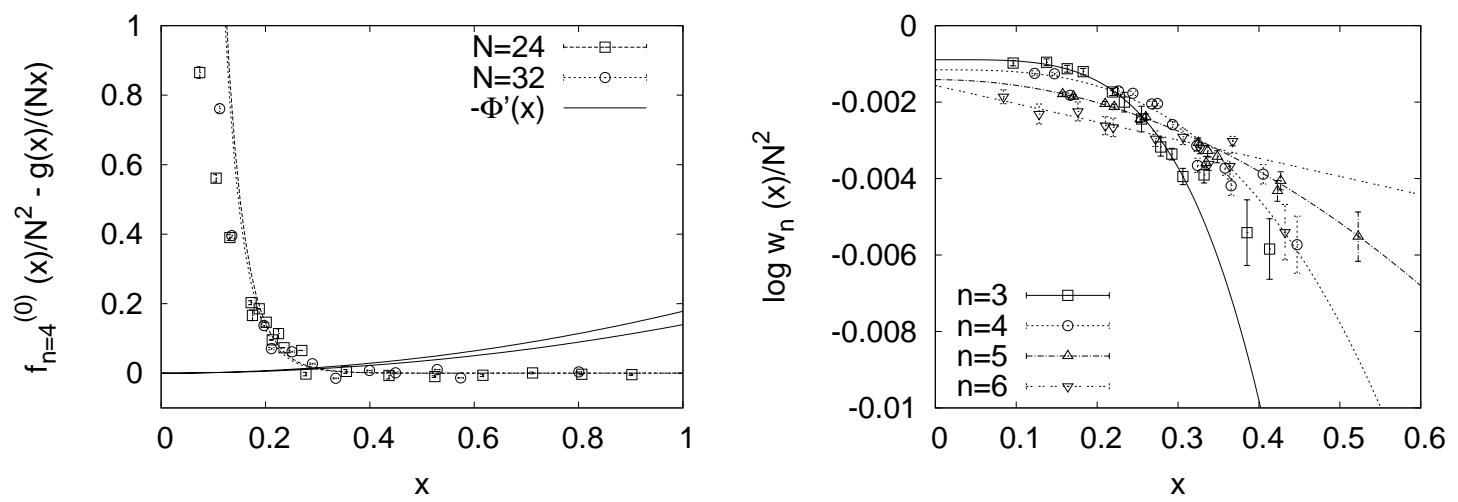

Figure 3: (LEFT) The solution of Eq. ([2.5), giving $\left\langle\tilde{\lambda}_{4}\right\rangle=0.31(2)$. The vertical axis and the corresponding fit of the data points estimate the LHS of (2.5]) in the large $N$ limit. (RIGHT) $\log w_{n}(x) / N^{2}$ for $N=24$ with the corresponding fits to their small $x$ scaling. These curves can be used to compute the difference $\Delta_{34}$ of Eq. (B.]).

at $x_{s} \approx 0.31$. The term $\int_{\mathrm{SO}(2)}^{\mathrm{SO}(3)} \frac{1}{N^{2}} \frac{d}{d x} \log \rho^{(0)}(x)$ vanishes in the large $N$ limit. The comparison of the free energies can be read off the right plot of Fig. B where the $N=24$ results are shown. Although we cannot draw a definite conclusion, we see no inconsistency with a dominating $\mathrm{SO}(3)$ vacuum as predicted by GEM.

In summary, we simulated from first principles a 6 dimensional version of the IIB matrix model. We studied the scenario of dynamical compactification by SSB of the eigenvalues of the tensor $T_{\mu \nu}$ and found that our results are consistent with the quantitative predictions of the GEM. The importance of the fluctuating phase in inducing SSB was demonstrated first by confirming that no SSB occurs in the phase quenched model. A strong complex action problem was found to hinder ordinary Monte Carlo simulations and the factorization method was used in order to determine and sample the region favored by the competing effects of the fluctuating phase, the real 
part of the action and the density of states. The distribution function $\rho_{n}(x)$ of the eigenvalues $\left\langle\tilde{\lambda}_{n}\right\rangle$ were considered and by using the scaling properties of the factors $f_{n}^{(0)}(x)$ and $w_{n}(x)$ we computed the scale $r$ of the compactified dimensions. The results were found to be consistent with the unique $d$-independent numerical value computed using the GEM and with the constant volume property $R^{d} r^{6-d}=\ell^{6}$. A strong, non perturbative, hard core potential against the collapse of the eigenvalues is shown to be generated dynamically which plays a crucial role in obtaining non trivial solutions in the large $N$ limit, generating scales $r$ and $R$ that are comparable and giving finite spacetime volume.

Recent results in [Q], however, have shown that it is possible to study the Lorentzian IIB matrix model and obtain an expanding, large, 3 dimensional space which arises after a critical time ${ }^{5}$. This is a very exciting possibility, which motivates further study of the IIB matrix model as a non perturbative definition of superstring theory and as a model for string inspired cosmology.

\section{References}

[1] N. Ishibashi, H. Kawai, Y. Kitazawa and A. Tsuchiya, Nucl. Phys. B 498, 467 (1997); H. Aoki, S. Iso, H. Kawai, Y. Kitazawa and T. Tada, Prog. Theor. Phys. 99 (1998) 713;for a review see e.g. H. Aoki et. al., Prog. Theor. Phys. Suppl. 134 (1999) 47; T. Azuma, arXiv:hep-th/0401120.

[2] J. Nishimura, G. Vernizzi, JHEP 0004, 015 (2000); Phys. Rev. Lett. 85, 4664-4667 (2000).

[3] J. Nishimura and F. Sugino, JHEP 0205 (2002) 001; H. Kawai et. al., Nucl. Phys. B 647, 153 (2002); Prog. Theor. Phys. 109, 115 (2003); T. Aoyama, J. Nishimura, T. Okubo, Prog. Theor. Phys. 125, 537-563 (2011); J. Nishimura, T. Okubo, F. Sugino, arXiv:1108.1293 [hep-th]; Prog. Theor. Phys. 114, 487 (2005).

[4] W. Krauth, H. Nicolai and M. Staudacher, Phys. Lett. B 431 (1998) 31; W. Krauth and M. Staudacher, Phys. Lett. B 435 (1998) 350; Phys. Lett. B 453 (1999) 253; T. Hotta, J. Nishimura and A. Tsuchiya, Nucl. Phys. B 545 (1999) 543.

[5] J. Ambjørn, K. N. Anagnostopoulos, W. Bietenholz, T. Hotta and J. Nishimura, JHEP 0007 (2000) 013; JHEP 0007 (2000) 011; J. Ambjørn, K. N. Anagnostopoulos, W. Bietenholz, F. Hofheinz and J. Nishimura, Phys. Rev. D 65 (2002) 086001;

[6] K.N. Anagnostopoulos, J. Nishimura, Phys. Rev. D 65, 105012 (2002).

[7] J. Ambjørn, K. N. Anagnostopoulos, J. Nishimura and J. J. M. Verbaarschot, JHEP 0210 (2002) 062; Phys. Rev. D 70 (2004) 035010; V. Azcoiti, G. Di Carlo, A. Galante and V. Laliena, Phys. Rev. Lett. 89 (2002) 141601; Z. Fodor, S. D. Katz and C. Schmidt, JHEP 0703 (2007) 121; S. Ejiri, Phys. Rev. D 77, 014508 (2008); K. N. Anagnostopoulos, T. Azuma, J. Nishimura, PoS LATTICE2010, 167 (2010).

[8] K.N. Anagnostopoulos, T. Azuma, J. Nishimura, Phys. Rev. D83, 054504 (2011); J. High Energy Phys. 10 (2011) 126.

[9] S. -W. Kim, J. Nishimura and A. Tsuchiya, Phys. Rev. Lett. 108 (2012) 011601; Phys. Rev. D 86 (2012) 027901; JHEP 1210 (2012) 147; PoS (Lattice2012) 230.

\footnotetext{
${ }^{5}$ In fact, it turned out that the complex action problem can be totally avoided in the Lorentzian model in a highly nontrivial manner [Q]
} 